\title{
Enhancing the Classical Closed-Loop Algorithm in terms of Power Consumption
}

\author{
Rahman Mirzaei $^{1,2}$ and Seyed Sina Kourehli ${ }^{3}$ \\ ${ }^{1}$ Department of Civil Engineering, Bonab Branch, Islamic Azad University, Bonab, Iran \\ ${ }^{2}$ The Research Center of Optimization and Engineering, Tabriz, Iran \\ ${ }^{3}$ Department of Civil Engineering, Ahar Branch, Islamic Azad University, Ahar, Iran
}

Correspondence should be addressed to Seyed Sina Kourehli; s-kourehli@iau-ahar.ac.ir

Received 25 September 2014; Accepted 1 February 2015

Academic Editor: Amir Hossein Gandomi

Copyright (C) R. Mirzaei and S. S. Kourehli. This is an open access article distributed under the Creative Commons Attribution License, which permits unrestricted use, distribution, and reproduction in any medium, provided the original work is properly cited.

An approach is suggested to reduce the peak and average control forces of actively controlled structures. In this method, responses of an actively controlled building should be much smaller than the responses of the same building controlled by the similar passive control mechanism. This approach leads to a time varying gain matrix, which is not restricted by external excitation but it is related to the selection of a scalar function. Extensive numerical analyses by using various scalar functions show that the proposed strategy effectively can reduce the need of the required control force consumptions.

\section{Introduction}

Significant efforts have been directed toward the possibility of employing protective systems in civil engineering structures including passive, semiactive, active, and hybrid control systems [1-5]. Among these, active control systems are the most advanced technique to achieve this issue. It makes a control mechanism act in a manner that automatically supplies the forces to control the responses of a structure depending upon the severity of the external forces and the instant state of the structure during vibrations. The direction and magnitude of the controlling forces in an active system are estimated by a competent algorithm. General concept of the modern control methods to reduce structural vibrations due to external excitations was proposed in the 1960s [6]. Thereafter, a vast amount of efforts has been devoted to develop a variety of control algorithms based on different control design criteria, like the classical optimal control method [7], the pole assignment method [8], the predictive control method [9], and the instantaneous optimal control method [10, 11]. Although the linear quadratic regulator algorithm, LQR [6], is known as the one of the most famous and historic methods with widespread applications in different fields (because of its simple procedure and ease of implementation on actual large-scale systems), only the classical closed-loop methods control is feasible for structural control applications. This means that there are some other methods which perform better than the closed-loop control algorithm; however, since they need foreknowledge of entire external disturbance time history, they are not applicable. On the other hand, since the Riccati equation is obtained by ignoring the earthquake excitation term, classical closed-loop control algorithms are approximately optimal methods and do not entirely satisfy the optimality conditions [12]. To overcome the shortcomings of the classical optimal control algorithms, several methods have been proposed until now such as the instantaneous optimal control method [10, 11], the generalized optimal active control (GOAC) [13], and the instantaneous optimal Wilson- $\theta$ method [11]. These algorithms, in spite of their suitable performances, are sensitive to change of time increment. Also, they could not significantly improve the performance of the controlled structure with respect to the classical optimal control methods. Basu and Nagarajaiah proposed a wavelet-based adaptive linear quadratic regulator formulation for optimal control problems [14]. They also presented a method for the control of time varying systems 
based on wavelet transformation [15]. Bagheri and Amini proposed a procedure based on pattern search method and wavelet to acquire a more efficient control scheme than the LQR [16].

In this paper, an approach called new linear quadratic regulator (NLQR) is proposed, in which a relationship is established between the active and passive structural responses, via a proper transformation matrix. Then, this relationship is linked to a scalar function (considering an additional assumption) which causes control force computations to be quite simple. In order to evaluate effectiveness and performance of the proposed method, it is applied to an 8-story shear type building structure to reduce its seismic vibration responses during the El-Centro earthquake excitation. A few proper scalar functions are derived using the El-Centro earthquake ground acceleration and then the performance of this building is evaluated during some other earthquake excitations.

\section{Classical Optimal Closed-Loop Algorithm (CCLQR)}

Consider a building equipped with an active control system excited by a strong ground motion. The governing dynamic equation of motion may be written as the following matrix form:

$$
M \ddot{x}+C \dot{x}+K x=-M E \ddot{x}_{g}+D u(t),
$$

where $x$ is the $n$-dimensional displacement vector and dots state the derivative of $x$ with respect to time as the velocity and acceleration vectors; $M, C$, and $K$ are the $n \times n$ mass, damping, and stiffness matrices of the structure, respectively, $E$ is the $n \times 1$ influence vector of the ground acceleration on the building masses, $D$ is the $n \times m$ location matrix of the control forces affecting the structure, and $u(t)$ is the $m \times 1$ control force vector applied by the $m$ actuators.

With some manipulations, the equation of motion may be rewritten in terms of the state-space variables $Z$ as follows:

$$
\dot{Z}=A Z(t)+B u(t)+H f(t), \quad Z\left(t_{0}\right)=Z_{0}
$$

in which $t_{0}$ is the initial time instant, $Z(t)$ is the vector of state variables, and $A$ depicts the system matrix, respectively. Vector $Z$ and matrix $A$ are defined as follows:

$$
Z(t)=[x(t), \dot{x}(t)], \quad A=\left[\begin{array}{cc}
0 & I \\
-M^{-1} K & -M^{-1} C
\end{array}\right] .
$$

In addition, matrix $B$ and vector $H$ are given as

$$
B=\left[\begin{array}{c}
0 \\
M^{-1} D
\end{array}\right], \quad H=\left[\begin{array}{c}
0 \\
-E
\end{array}\right] .
$$

In the classical linear optimal control, a performance index $J(t)$ is defined in order to minimize control forces to achieve the best structural performances as

$$
J=\int_{0}^{t f}\left[Z^{T}(t) Q Z(t)+u^{T}(t) R u(t)\right] d t,
$$

where $Q$ is a $2 n \times 2 n$ positive semidefinite weighting matrix related to the structural response, $R$ is an $r \times r$ positive definite weighting matrix related to the active control force, and $t_{f}$ indicates the terminal time that should be longer than the earthquake duration.

In classical closed-loop quadratic regulator (CCLQR), optimal control force is achieved by minimizing a performance index $J(t)$ with respect to the vectors $Z(t)$ and $u(t)$ as follows:

$$
u(t)=-\frac{1}{2} R^{-1} B^{T} P(t) Z(t),
$$

where $P(t)$ is the Riccati matrix, obtained by solving the following nonlinear matrix equation:

$$
\begin{aligned}
& {\left[\dot{P}(t)+P(t) A-\frac{1}{2} P(t) B R^{-1} B^{T} P(t)+A^{T} P(t)+2 Q\right] Z(t)} \\
& \quad+P(t) H f(t)=0, \quad P\left(t_{f}\right)=0 .
\end{aligned}
$$

There are two assumptions to solve (7); first, the external disturbance $f(t)$ is set to zero (it is assumed as a stochastic white noise process); second, the Riccati matrix is a constant one over the time. Although the second assumption is almost acceptable, the first assumption is not fulfilled in all situations. Anyway, (7) can be rewritten as the following one:

$$
P A-\frac{1}{2} P B R^{-1} B^{T} P+A^{T} P+2 Q=0 .
$$

By selecting appropriate $Q$ and $R$ weighting matrices, this equation can simply be solved and, according to (6), the constant gain matrix is determined as

$$
G=-\frac{1}{2} R^{-1} B^{T} P .
$$

\section{Proposed Linear Quadratic Regulator (NLQR)}

As we know, by removing control forces of active mass drivers (AMDs), an active control system may passively perform as tuned mass dampers (TMDs). Hence, from control mechanisms point of view, first we assume that the building is controlled by a virtual passive controller which includes a few single degree of freedom systems as TMDs tuned to the building. Then, by employing the similar active control system, in which TMDs were replaced by AMDs, control forces are determined in the way that the responses of the active controlled building are a portion of the similar responses in the passively controlled building. The new proposed method or the new linear quadratic regulator (NLQR) is formed based on this idea. In the first case, we assume that the dynamic equation of motion of the passively controlled building in terms of the state-space variables, shown by $\bar{Z}$, is

$$
\dot{\bar{Z}}=A \bar{Z}(t)+H f(t), \quad \bar{Z}\left(t_{0}\right)=0 .
$$

Now, suppose that in practice the building is actively controlled by AMDs with the same location and characteristics of 
the passive control system. AMDs apply active control forces to the building such that the building responses are much reduced with respect to the structural responses of the first case, as

$$
Z(t)=T(t) \bar{Z}(t),
$$

where $T(t)$ is an admissible transformation matrix. It should be mentioned that the passive control system is a virtual system; therefore, there is no need to know the responses of the passively controlled building. In other words, the transformation matrix is not tuned for a specific external excitation, but it is considered based on the building properties in a way where the building performs better against external disturbances. By using first derivative of (11) with respect to time, we have

$$
\dot{Z}(t)=\dot{T}(t) \bar{Z}(t)+T(t) \dot{\bar{Z}}(t) .
$$

By combining (2) and (10) in (12) and using the transform relation of (11) and (6), after some manipulations, (13) is obtained:

$$
\begin{aligned}
& \left(\dot{T}(t)-A T(t)+T(t) A+\frac{1}{2} B R^{-1} B^{T} P T(t)\right) \bar{Z}(t) \\
& \quad=(I-T(t)) H f(t) .
\end{aligned}
$$

For further convenience, assume that the transform matrix of (11) is chosen as an identity matrix premultiplied by a scalar time dependent function as follows:

$$
T(t)=\alpha(t) \cdot I, \quad \dot{T}(t)=\dot{\alpha}(t) \cdot I .
$$

Then, the external excitation term in (13) is directly related to the passive state-space variable through a simple form:

$$
\begin{aligned}
& \left(\dot{\alpha}(t) \cdot I+\frac{\alpha(t)}{2} B R^{-1} B^{T} P\right) \bar{Z}(t)=(1-\alpha(t)) H f(t) \quad \text { or } \\
& \left(\frac{\dot{\alpha}(t)}{(1-\alpha(t))} \cdot I+\frac{\alpha(t)}{2(1-\alpha(t))} B R^{-1} B^{T} P\right) \bar{Z}(t)=H f(t) .
\end{aligned}
$$

This equation represents the relation between external excitation and passively controlled responses of the structure. By inserting (15) in optimization process of (7) and using (11), as well as ignoring the first derivative of $P$ matrix, the following equation is obtained:

$$
\begin{aligned}
& P\left(A+\left(\frac{\dot{\alpha}(t)}{2 \alpha(t)(1-\alpha(t))}\right) I\right) \\
& \quad-\frac{1}{2} P B\left(-\left(\frac{1-\alpha(t)}{\alpha(t)}\right) \cdot R\right)^{-1} B^{T} P \\
& \quad+\left(A+\left(\frac{\dot{\alpha}(t)}{2 \alpha(t)(1-\alpha(t))}\right) I\right)^{T} P+2 Q=0 .
\end{aligned}
$$

This equation is a modified form of (8), in which matrices $A$ and $R$ are changed based on the selected scalar function, $\alpha(t)$. Now, by proper adjustment of the scalar function, $\alpha(t)$, and solving (16), time dependent gain matrix is achieved as

$$
G(t)=-\frac{1}{2} R^{-1} B^{T} P(t) .
$$

\section{Limitations of the Scalar Function $\alpha(t)$}

With a glance at (16), we find out that the proposed method modifies definition of matrices $A$ and $R$ in (8) to the new forms as follows:

$$
\begin{gathered}
A \longrightarrow A+\lambda(t) I=A+\left(\frac{\dot{\alpha}(t)}{2 \alpha(t)(1-\alpha(t))}\right) I, \\
R \longrightarrow \gamma(t) R=-\left(\frac{1-\alpha(t)}{\alpha(t)}\right) \cdot R .
\end{gathered}
$$

Equation (18) dictates that the scalar function, $\alpha(t)$, has to satisfy the following two important criteria: (1) we know that, based on the Lyapunov stability criteria in the continuoustime state-space formulation, an active controlled building will be stable if the eigenvalues of its system matrix have negative real parts. On the other hand, according to the $\alpha$ shift method, by using $A+\lambda(t) I$, we forced our system to have eigenvalues with real parts less than $-\lambda(t)$. Hence, if the coefficient of identity matrix, $\lambda(t)$, added to $A$ in (18) is positive, stability of the whole system will be ensured during control time; (2) $R$ matrix is a positive definite matrix. Necessarily, therefore, the coefficient of $\gamma(t)$ in (18) should be positive over the time to satisfy this criterion.

\section{Numerical Example}

In order to compare performance of the proposed method (NLQR) in comparison with the classical closed-loop optimal algorithm (CCLQR), an eight-story shear type building is considered. All the properties of the floors are identical; the floor mass of each story is equal to 345.5 tons, the elastic stiffness of each story is $3.404 \times 10^{5} \mathrm{kN} / \mathrm{m}$, and the internal damping capacity of each story is 2937 tons-sec/m. The active control system includes an active mass damper/driver (AMD) mechanism, which is installed at the roof. The characteristics of AMD are as follows: its mass is 29.63 tons, its tuned frequency is $98 \%$ of the predominant vibration frequency of the building without control, and its damping is 25 tons$\mathrm{sec} / \mathrm{m}$.

5.1. Ground Accelerations. Performance of the active controlled building is considered during four different ground motions, which include the El-Centro earthquake, the Hyogoken-Nanbu (Kobe) earthquake, the Landers earthquake, and the Parkfield earthquake. These acceleration time histories are shown in Figures 1 to 4.

The active control system is designed such that the controlled building presents the best performance during the El-Centro earthquake excitation. Then, behavior of the controlled building during other earthquake excitations is evaluated and their performances are compared together.

5.2. Selection of a Proper Scalar Function $\alpha(t)$. Based on Section 4, three different functions are presented here as suitable options for the scalar function. The first scalar function is as follows:

$$
\alpha_{1}(t)=1+e^{\beta t}, \quad \beta \in R
$$




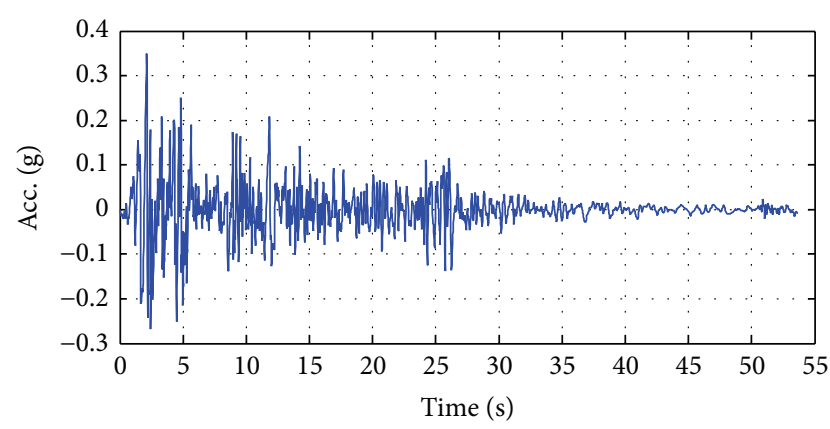

FIGURE 1: Time history of El-Centro earthquake.

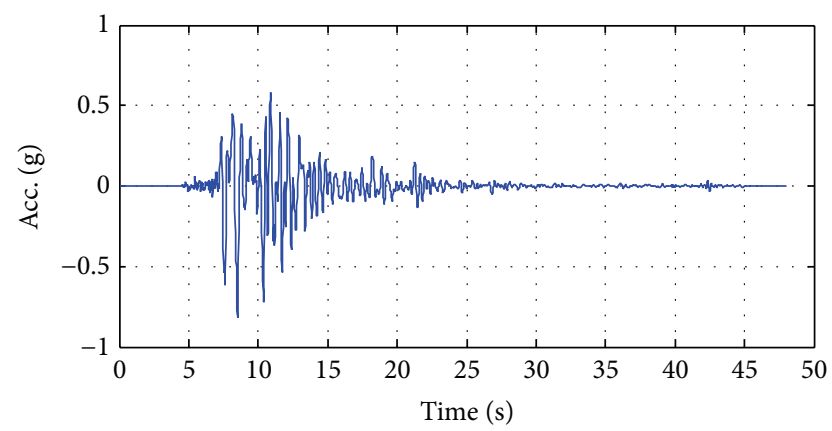

FIgURE 2: Time history of Hyogoken-Nanbu earthquake.

This function is arbitrarily selected to achieve desirable performances against the El-Centro ground motion. Appropriate value for variable $\beta$ is equal to 2 . follows:

As a second case, another scalar function is proposed as

$$
\alpha_{2}(t)=1+e^{-\beta\left(t-t_{o}\right)^{2}}, \quad t_{o} \gg t_{f}, \beta \in R .
$$

Appropriate value for variable $\beta$ is set to 0.001 . Parameter $t_{0}$ is an auxiliary variable to set the necessary conditions presented in Section 3, which should be much longer than the length of the control time duration. As a rule, $t_{0}$ may be almost two or three times greater than the control time.

Different than the above functions, the last scalar function is proposed as an acceleration time history dependent function. This function uses time history of the recorded earthquake until that time to find the instantaneous Riccati matrix in each time instant. The proposed function is as follows:

$$
\begin{aligned}
& \alpha_{3}(t)=e^{-\eta t}\left(1+e^{-f(t)}\right) \\
& \text { where } f(t)=\left\{\int_{0}^{t}\left|\frac{\ddot{x}_{g}}{g}\right| d t\right\}^{n} \quad \eta \in R^{+}, \quad n \in R .
\end{aligned}
$$

After extensive analysis, the appropriate values for variables $\eta$ and $n$ in (21) are set to be 0.1 and 1.5, respectively. This function satisfies the necessary conditions of Section 4 but as it was previously mentioned, the rates of change of the two variables $\lambda(t)$ and $\gamma(t)$ are very rapid. Hence, the Riccati

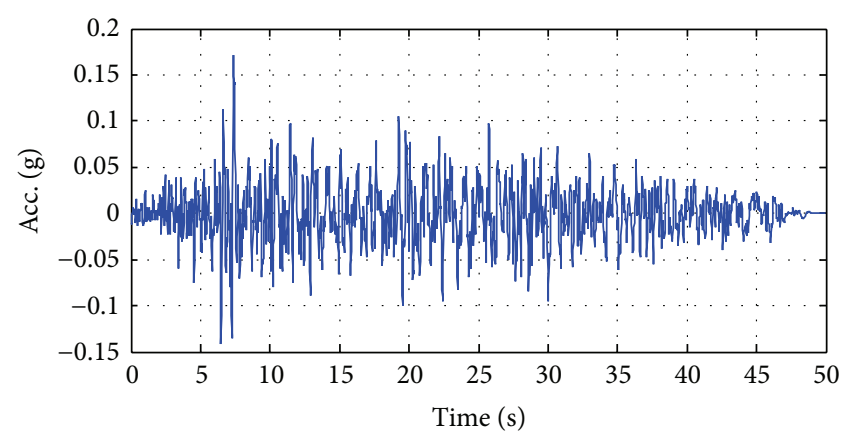

Figure 3: Time history of Landers earthquake.

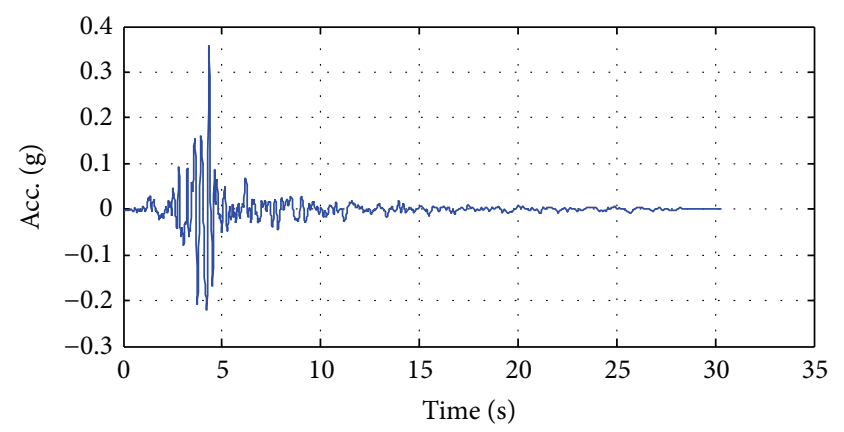

Figure 4: Time history of Parkfield earthquake.

matrix independent of the previous and the next time instant should be found.

5.3. Evaluation Criteria. To evaluate the efficiency of control algorithms, researchers have employed various indices such as the maximum displacement, velocity, or acceleration of the stories, the drift ratios of the adjacent floors, and the maximum base shear of the structures. In this paper, different criteria are developed in order to painstakingly compare the efficiency of different algorithms. Table 1 summarized these categories of criteria, in which indices $J_{1}$ through $J_{3}$ represent the criteria for the maximum displacement, velocity, and acceleration responses of the top story, which are normalized to their corresponding uncontrolled values, that is, the structure without any active or passive control systems.

The performance index $J_{4}$ represents the normalized maximum base shear of the controlled building with respect to the uncontrolled one. Indices $J_{5}$ through $J_{8}$ show the root mean square (RMS) of the maximum story responses, such as the displacement, velocity, acceleration, and the story drifts, with respect to the corresponding response quantities in the uncontrolled case. Finally, indices $J_{9}$ and $J_{10}$ represent the maximum and average amounts of the required control forces with respect to the classical closedloop optimal control algorithm. Meanwhile, an additional parameter, called efficiency index (E.I.), is defined as the average of the indices $J_{1}$ through $J_{8}$. All of these indices help to show an overall insight on the performances of the various control systems. 
TABle 1: Performance indices.

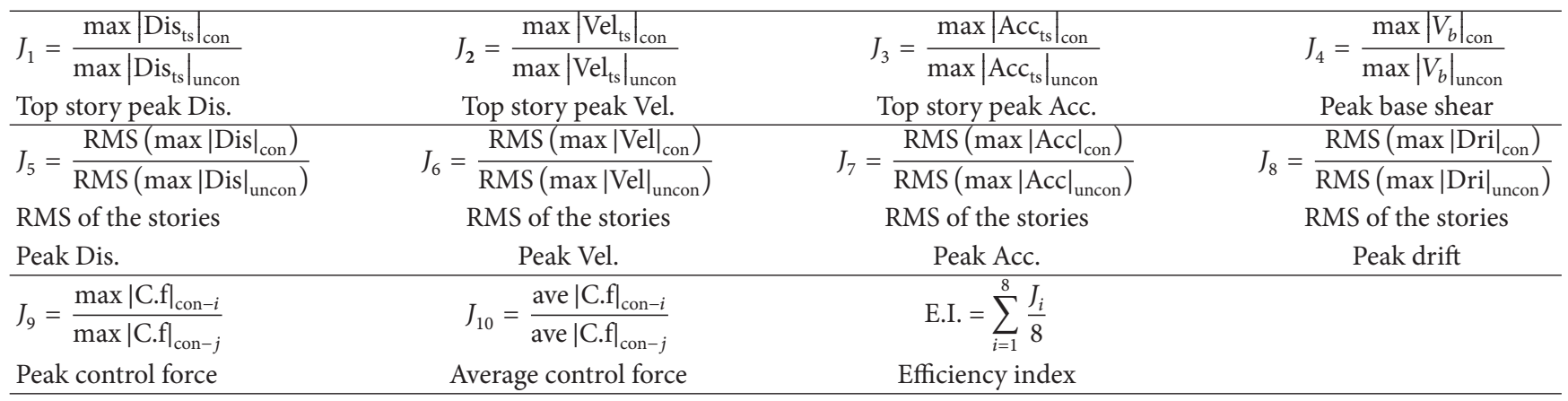

5.4. Selection of Weighting Matrices. In many researches [17], variety of $Q$ weighting matrix has been suggested. Proper arrangement of the weighting matrix elements and increasing or decreasing its values may significantly affect the stability of the controlled structure and the performance of the control system to achieve the objectives. In order to investigate the performance of the proposed method using various scalar functions, it is assumed that the arrangements of the $Q$ weighting matrix are the same for the proposed method and the classical optimal algorithm:

$$
Q=1 \times 10^{4}\left[\begin{array}{cc}
K & 0 \\
0 & M
\end{array}\right],
$$

where $K$ and $M$ are the matrices, with dimensionless numerical values corresponding to the stiffness and mass matrices of the controlled building, omitting the stiffness and mass values of the active mass damper/driver. Weighting matrix related to the control force, $R$, is assigned to be equal to 1 for all algorithms. Notice that matrix $R$ is a scalar quantity, because only one AMD is installed at the roof level.

\subsection{Stability Diagram of the Actively Controlled Building.} Since the proposed method results in a time varying gain matrix, stability of the whole controlled building may be changed over the time. In order to inspect this issue, stability diagrams of the building equipped with active and passive systems are depicted together in Figures 5 to 7. Stability diagram of the building, using scalar function $\alpha_{1}(t)$, is plotted in Figure 5. At the beginning of ground motion the structure is more stable while as time goes away poles of actively controlled building move toward passively controlled building poles. On the other hand, in Figure 6, the stability diagram for the case corresponding to scalar function $\alpha_{2}(t)$ shows a reverse treatment in comparison with scalar function $\alpha_{1}(t)$. Finally, in Figure 7, the stability diagram of the controlled building using $\alpha_{3}(t)$ is depicted. In this figure, a manner similar to case one can be seen.

5.6. Response of the Controlled Building during the El-Centro Earthquake Excitation. In order to examine performance of the proposed control method, linear time history analysis is carried out for all mentioned cases. Maximum acceleration, velocity, and displacement responses of all floors including

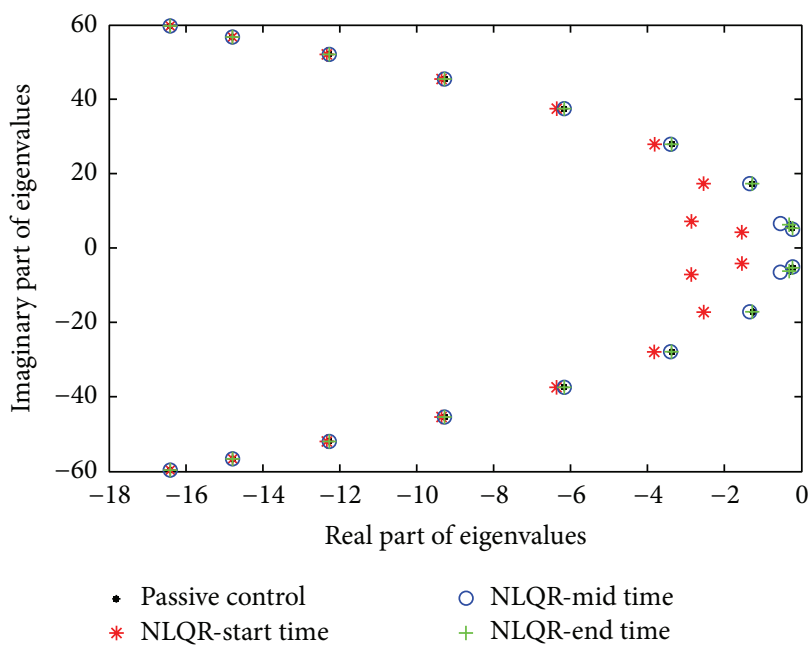

FIGURE 5: Stability diagram of the controlled building using $\alpha_{1}(t)$ scalar function, the El-Centro earthquake.

passive and active control systems using CCLQR and NLQR with different scenarios during the El-Centro earthquake excitation are compared with the responses of the uncontrolled building as shown in Figure 8 in which the proposed method using various scenarios can significantly decrease all responses of the floors. The best responses belong to the third scalar function $\alpha_{3}(t)$, which is almost the same as the CCLQR algorithm.

Numerical results of the responses of the controlled building are briefly tabulated in Table 2 . The maximum and the root mean square of the top story responses, maximum and average required control force, maximum values of the base shear, and the root mean square of the floor drifts for different control systems are presented. Comparing the results shows that the best performance belongs to CCLQR and the results of the new proposed method using the third scalar function $\alpha_{3}(t)$ are also very close to it. The only difference is in consuming control forces, which may be observed in columns 8 and 9 of Table 2. Decreasing in maximum control force is only about $1.2 \%$ but decreasing in the average (or total) required control force is about $28 \%$. This is the major capability of the proposed method. It is noted 
TABLE 2: Structural peak response of the controlled building during the El-Centro earthquake excitation.

\begin{tabular}{lcccccccccc}
\hline Case & $\begin{array}{c}\text { Top story } \\
\text { Dis. }(\mathrm{cm})\end{array}$ & $\begin{array}{c}\text { Top story } \\
\text { Vel. }(\mathrm{m} / \mathrm{s})\end{array}$ & $\begin{array}{c}\text { Top story } \\
\text { Acc. }\left(\mathrm{m} / \mathrm{s}^{2}\right)\end{array}$ & $\begin{array}{c}\text { RMS of } \\
\text { Dis. }(\mathrm{cm})\end{array}$ & $\begin{array}{c}\text { RMS of } \\
\text { Vel. }(\mathrm{m} / \mathrm{s})\end{array}$ & $\begin{array}{c}\text { RMS of } \\
\text { Acc. }\left(\mathrm{m} / \mathrm{s}^{2}\right)\end{array}$ & $\begin{array}{c}\text { Max. Co.f. } \\
(\mathrm{KN})\end{array}$ & $\begin{array}{c}\text { Ave. Co.f. } \\
(\mathrm{KN})\end{array}$ & $\begin{array}{c}\text { Max. B. } \\
\text { shear }(\mathrm{KN})\end{array}$ & $\begin{array}{c}\text { RMS of } \\
\mathrm{drifts}(\mathrm{cm})\end{array}$ \\
\hline No control & 19.3 & 1.06 & 7.9 & 39.16 & 2.23 & 15.68 & - & - & 11.07 & 7.34 \\
Passive control & 15.2 & 0.89 & 6.4 & 31.09 & 1.84 & 13.13 & - & - & 9.24 & 5.87 \\
CCLQR & 6.70 & 0.63 & 3.60 & 13.74 & 1.41 & 8.92 & 1082.82 & 119.84 & 4.08 & 2.74 \\
NLQR- $\alpha_{1}(t)$ & 8.90 & 0.68 & 4.60 & 18.34 & 1.52 & 10.09 & 763.15 & 83.25 & 5.49 & 3.42 \\
NLQR- $\alpha_{2}(t)$ & 7.80 & 0.68 & 4.40 & 16.10 & 1.49 & 9.40 & 739.79 & 99.17 & 4.86 & 3.12 \\
NLQR- $\alpha_{3}(t)$ & 6.80 & 0.63 & 4.10 & 14.07 & 1.44 & 9.25 & 1069.47 & 86.00 & 4.17 & 2.77 \\
\hline
\end{tabular}

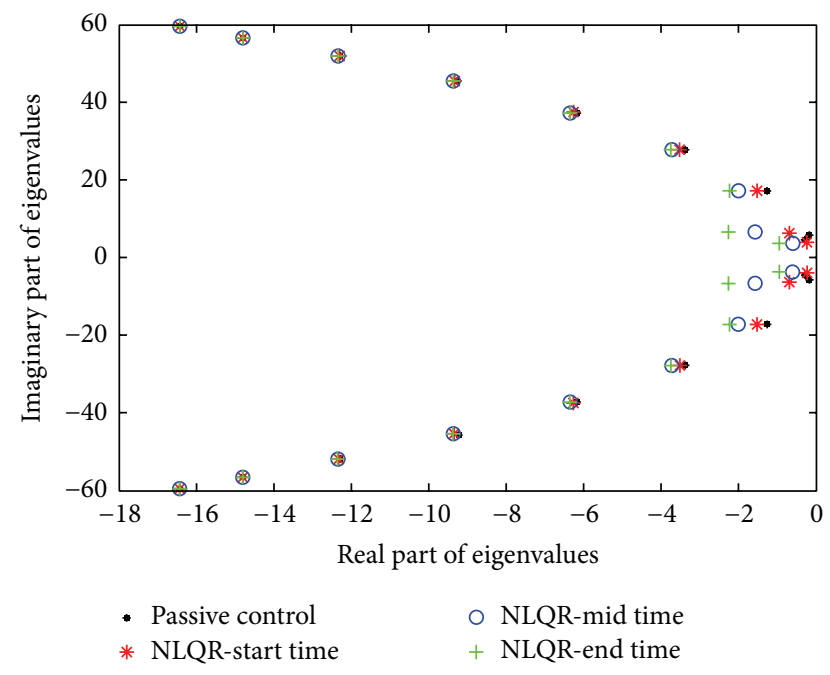

FIGURE 6: Stability diagram of the controlled building using $\alpha_{2}(t)$ scalar function, the El-Centro earthquake.

that selecting proper scalar function plays an important role in decreasing the need of the total power supply of the system.

5.7. Active Control Forces against the El-Centro Earthquake Excitation. Active control force time histories of the proposed method using three various scalar functions are compared to that of CCLQR as shown in Figures 9 and 10. In Figure 9, it is observed that the control system using the first scalar function $\alpha_{1}(t)$ totally determines active control forces lower than CCLQR. Figure 10 shows that at the start time of control duration the system using the second scalar function $\alpha_{2}(t)$ determines active control forces lower than CCLQR, but after a while by changing its poles, control forces are increased. Therefore, it is expected that the performance of the control system using $\alpha_{2}(t)$ is better than the previous one but not as good as CCLQR.

Figure 11 shows that the control system using the third scalar function requires the same control forces as CCLQR when the acceleration ground motion is large. Additionally, as the severity of the earthquake is decreased, the control force demand decreases too.

Briefly regarding the above discussion, we can say NLQR with $\alpha_{3}(t)$ may present the best performance of the control

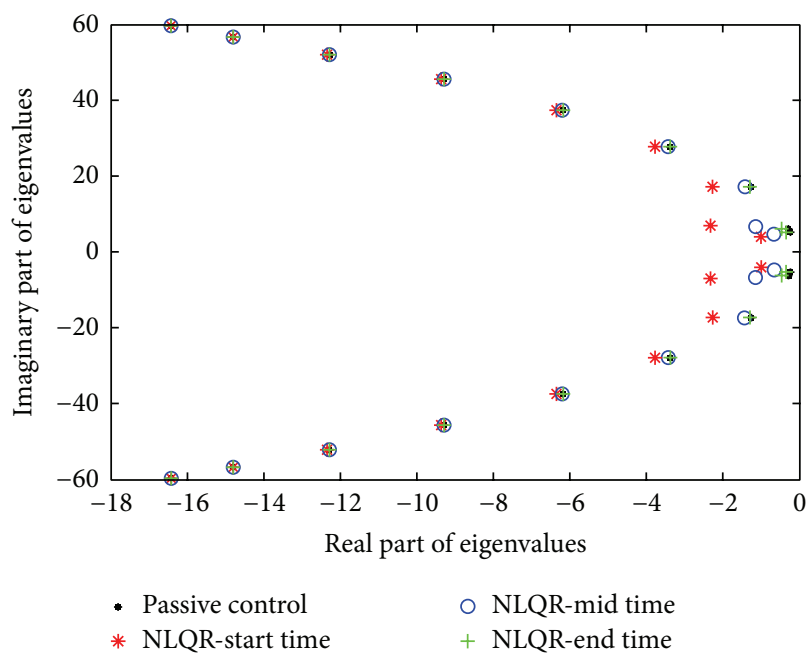

FIGURE 7: Stability diagram of the controlled building using $\alpha_{3}(t)$ scalar function, the El-Centro earthquake.

system since instant control force follows the severity of the ground excitations.

5.8. Response of the Controlled Building during the Other Earthquake Excitations. Performances of various control systems, which are designed to achieve the high efficiency during the El-Centro earthquake, are examined under a few other earthquakes excitations including the HyogokenNanbu earthquake, the Landers earthquake, and the Parkfield earthquake. After computing, performance indices of the controlled building for each earthquake are listed in Tables 3 to 5 . The indices of controlled building using NLQR- $\alpha_{1}(t)$ show the lowest efficiency in comparison with the other cases. High values of its separate indices or its averaged index, E.I., confirm this statement.

Generally, the control system using $\alpha_{2}(t)$ presents the middle performance between the three scalar functions, except the Landers earthquake, in which the performance is better than NLQR- $\alpha_{3}(t)$. By noticing the earthquake records in Figures 1 to 4 , it is seen that the strong part of the Landers record is distributed over the whole duration of the signal, while for the other earthquakes it is concentrated at a short length beyond the beginning. Since NLQR- $\alpha_{2}(t)$ increases 


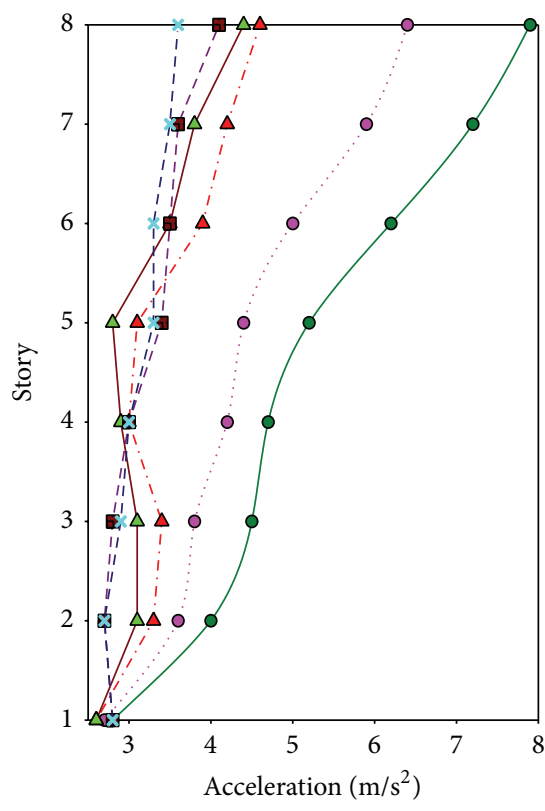

- Without control $\triangle$ NLQR- $\alpha(t)=\alpha_{2}$

o. Passive control $-\mathbf{a}-\mathrm{NLQR}-\alpha(t)=\alpha_{3}$

$\Delta \operatorname{NLQR}-\alpha(t)=\alpha_{1}-\approx-\operatorname{CCLQR}$

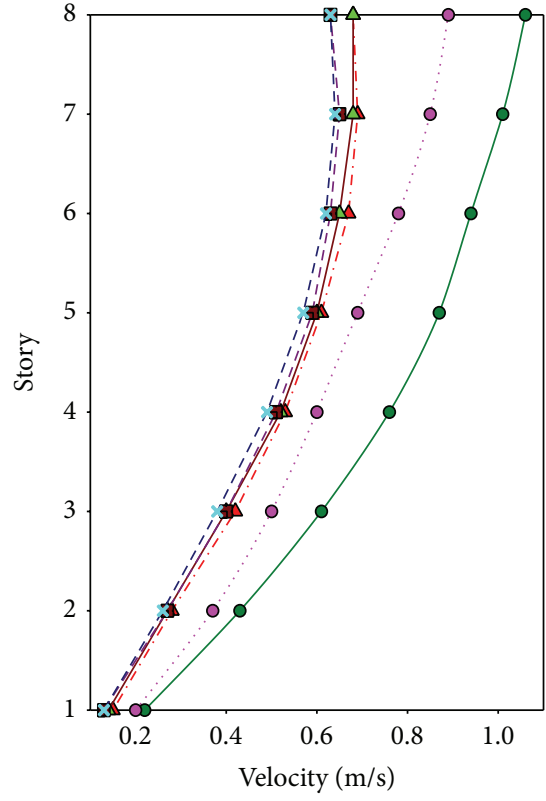

- Without control $\triangle$ NLQR- $\alpha(t)=\alpha_{2}$

-.. Passive control - - NLQR- $\alpha(t)=\alpha_{3}$

$\Delta \operatorname{NLQR}-\alpha(t)=\alpha_{1}-*-\operatorname{CCLQR}$

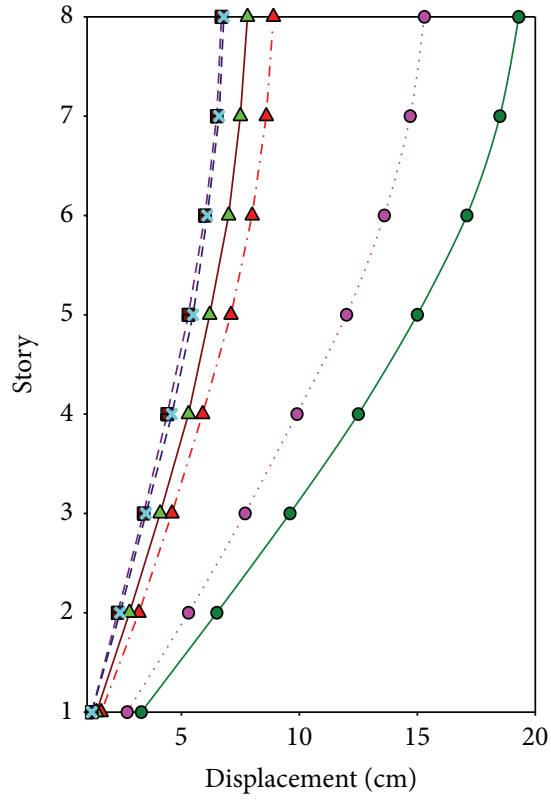

- Without control $\triangle \mathrm{NLQR}-\alpha(t)=\alpha_{2}$

-. Passive control - - NLQR- $\alpha(t)=\alpha_{3}$

$\Delta \operatorname{NLQR}-\alpha(t)=\alpha_{1}-x-\operatorname{CCLQR}$

FIGURE 8: Maximum responses of the floors, comparing active, passive, and uncontrolled cases, when the structure is subjected to the ElCentro earthquake excitation.

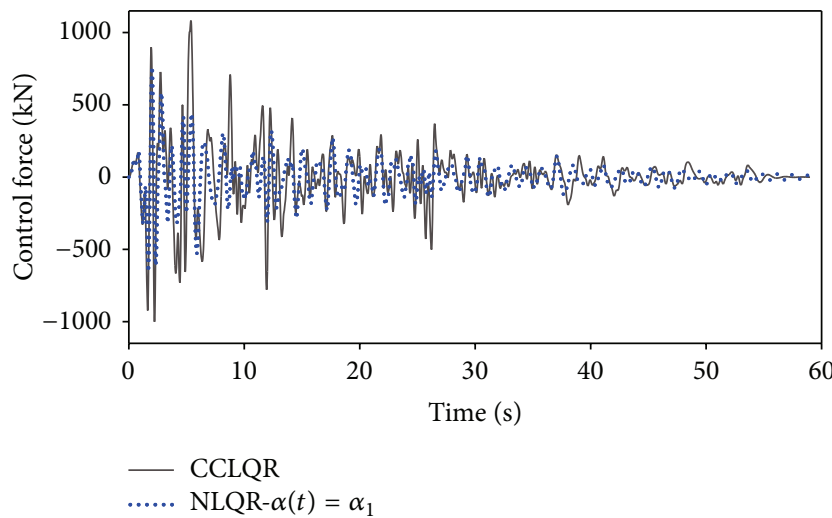

FIGURE 9: Control force time history of NLQR using $\alpha_{1}(t)$ scalar function, during the El-Centro earthquake excitation.

control force with time, it is expected to act more effective than the other scalar functions in this case.

NLQR- $\alpha_{3}(t)$ in almost all cases gives satisfactory performances close to the CCLQR, with lower maximum and average control forces demand. This may play a crucial role when severe earthquakes happen.

The average values of the results obtained from all earthquakes are summarized in Table 6. As can be seen, the proposed control method using various scalar functions may generally reduce responses as well as both the maximum and the average required control forces of the building much better than a similar control system. Extensive results during

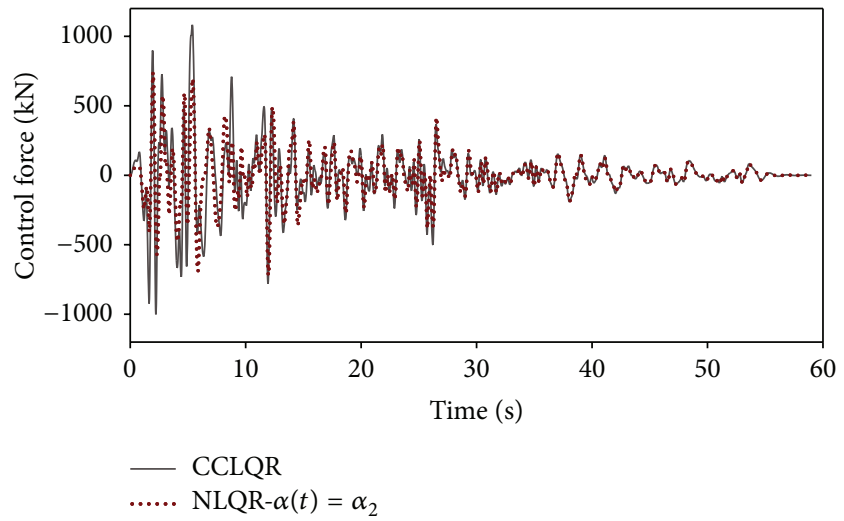

FIGURE 10: Control force time history of NLQR using $\alpha_{2}(t)$ scalar function, during the El-Centro earthquake excitation.

different strong shakings show that high efficiency of NLQR is obtained when a scalar function related to the external excitation or combination of two dissimilar functions, like the second and the third scalar functions, is employed.

\section{Conclusion}

In this paper, an approach, called NLQR, is suggested for reducing the peak and average required control forces in actively controlled structures employing optimal control methods. In order to evaluate the effectiveness of the proposed method, the responses of an 8-story shear type 


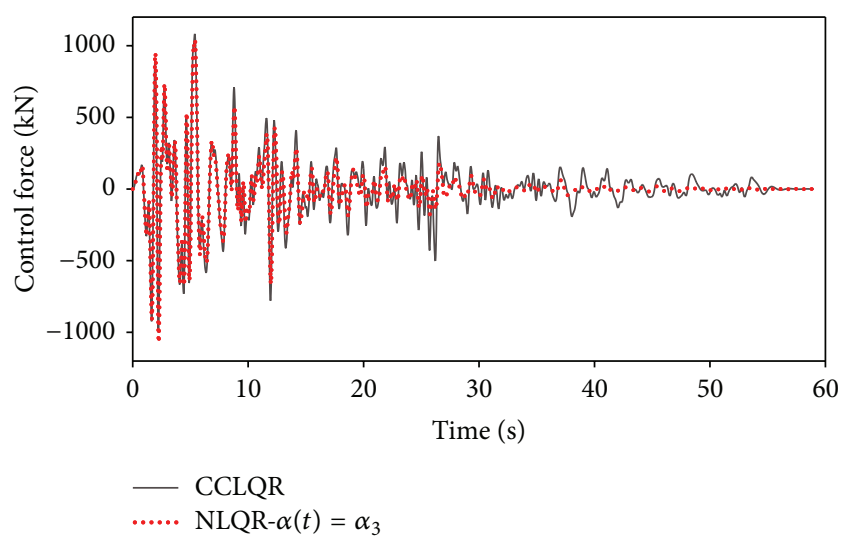

FIGURE 11: Control force time history of NLQR using $\alpha_{3}(t)$ scalar function, during the El-Centro earthquake excitation.

TABLE 3: Performance indices for building, subjected to HyogokenNanbu earthquake excitation.

\begin{tabular}{lccccc}
\hline Index & $\begin{array}{c}\text { Passive } \\
\text { control }\end{array}$ & CCLQR NLQR- $\alpha_{1}(t)$ & NLQR- $\alpha_{2}(t)$ & NLQR- $\alpha_{3}(t)$ \\
\hline$J_{1}$ & 0.94 & 0.50 & 0.74 & 0.57 & 0.55 \\
$J_{2}$ & 0.96 & 0.60 & 0.75 & 0.66 & 0.65 \\
$J_{3}$ & 0.96 & 0.55 & 0.88 & 0.68 & 0.66 \\
$J_{4}$ & 0.94 & 0.51 & 0.84 & 0.62 & 0.56 \\
$J_{5}$ & 0.94 & 0.51 & 0.74 & 0.57 & 0.56 \\
$J_{6}$ & 0.96 & 0.61 & 0.76 & 0.67 & 0.66 \\
$J_{7}$ & 0.95 & 0.73 & 0.97 & 0.83 & 0.79 \\
$J_{8}$ & 0.94 & 0.54 & 0.81 & 0.63 & 0.58 \\
\hline E.I. & - & 0.57 & 0.81 & 0.65 & 0.63 \\
\hline$J_{9}$ & - & 1 & 0.66 & 0.94 & 0.89 \\
$J_{10}$ & - & 1 & 0.95 & 0.92 & 0.80 \\
\hline
\end{tabular}

TABle 4: Performance indices for building, subjected to Landers ground excitation.

\begin{tabular}{lccccc}
\hline Index & $\begin{array}{c}\text { Passive } \\
\text { control }\end{array}$ & CCLQR & NLQR- $\alpha_{1}(t)$ & NLQR- $\alpha_{2}(t)$ & NLQR- $\alpha_{3}(t)$ \\
\hline$J_{1}$ & 0.78 & 0.34 & 0.60 & 0.42 & 0.51 \\
$J_{2}$ & 0.78 & 0.44 & 0.56 & 0.51 & 0.47 \\
$J_{3}$ & 0.97 & 0.65 & 0.87 & 0.81 & 0.77 \\
$J_{4}$ & 0.74 & 0.34 & 0.54 & 0.40 & 0.50 \\
$J_{5}$ & 0.77 & 0.33 & 0.57 & 0.41 & 0.49 \\
$J_{6}$ & 0.85 & 0.51 & 0.59 & 0.52 & 0.52 \\
$J_{7}$ & 0.89 & 0.66 & 0.77 & 0.71 & 0.68 \\
$J_{8}$ & 0.78 & 0.37 & 0.60 & 0.43 & 0.52 \\
\hline E.I. & - & 0.45 & 0.64 & 0.53 & 0.56 \\
\hline$J_{9}$ & - & 1 & 0.54 & 0.79 & 0.85 \\
$J_{10}$ & - & 1 & 0.77 & 0.96 & 0.54 \\
\hline
\end{tabular}

building structure subjected to different ground motions are evaluated. Different types of scalar functions are also utilized for this purpose. The extensive analyses show that NLQR can decrease all responses of the building similar to CCLQR
TABle 5: Performance indices for building, subjected to Parkfield ground excitation.

\begin{tabular}{lccccc}
\hline Index & $\begin{array}{c}\text { Passive } \\
\text { control }\end{array}$ & CCLQR & NLQR- $\alpha_{1}(t)$ & NLQR- $\alpha_{2}(t)$ & NLQR- $\alpha_{3}(t)$ \\
\hline$J_{1}$ & 0.89 & 0.35 & 0.52 & 0.40 & 0.37 \\
$J_{2}$ & 0.95 & 0.60 & 0.78 & 0.72 & 0.62 \\
$J_{3}$ & 0.93 & 0.50 & 0.72 & 0.67 & 0.55 \\
$J_{4}$ & 0.85 & 0.47 & 0.54 & 0.45 & 0.46 \\
$J_{5}$ & 0.88 & 0.40 & 0.50 & 0.40 & 0.41 \\
$J_{6}$ & 0.92 & 0.62 & 0.71 & 0.65 & 0.62 \\
$J_{7}$ & 0.95 & 0.64 & 0.81 & 0.73 & 0.65 \\
$J_{8}$ & 0.89 & 0.48 & 0.60 & 0.50 & 0.48 \\
\hline E.I. & - & 0.51 & 0.65 & 0.57 & 0.52 \\
\hline$J_{9}$ & - & 1 & 0.23 & 0.55 & 0.92 \\
$J_{10}$ & - & 1 & 0.45 & 0.78 & 0.81 \\
\hline
\end{tabular}

TABLE 6: Averaged performance indices of the controlled building, subjected to the El-Centro, Hyogoken-Nanbu, Landers, and Parkfield earthquakes.

\begin{tabular}{lccccc}
\hline Index & $\begin{array}{c}\text { Passive } \\
\text { control }\end{array}$ & CCLQR NLQR- $\alpha_{1}(t)$ & NLQR- $\alpha_{2}(t)$ & NLQR- $\alpha_{3}(t)$ \\
\hline$J_{1}$ & 0.85 & 0.39 & 0.58 & 0.45 & 0.45 \\
$J_{2}$ & 0.88 & 0.56 & 0.68 & 0.63 & 0.58 \\
$J_{3}$ & 0.92 & 0.54 & 0.76 & 0.68 & 0.63 \\
$J_{4}$ & 0.84 & 0.42 & 0.60 & 0.48 & 0.48 \\
$J_{5}$ & 0.85 & 0.40 & 0.57 & 0.45 & 0.45 \\
$J_{6}$ & 0.89 & 0.60 & 0.69 & 0.63 & 0.61 \\
$J_{7}$ & 0.91 & 0.65 & 0.80 & 0.72 & 0.68 \\
$J_{8}$ & 0.86 & 0.44 & 0.62 & 0.49 & 0.49 \\
\hline E.I & - & 0.50 & 0.66 & 0.57 & 0.55 \\
\hline$J_{9}$ & - & 1 & 0.53 & 0.74 & 0.91 \\
$J_{10}$ & - & 1 & 0.72 & 0.87 & 0.72 \\
\hline
\end{tabular}

but almost in all cases it effectively reduces the need for the required control force consumptions.

\section{Conflict of Interests}

The authors declare that there is no conflict of interests regarding the publication of this paper.

\section{References}

[1] N. M. Rahbari and S. Talatahari, "On the efficiency of semiactive smart structures: self-regulating MR dampers control system for tall buildings," The Structural Design of Tall and Special Buildings, vol. 23, no. 13, pp. 1027-1044, 2014.

[2] X. Zhang and F. Y. Cheng, "Control force estimation in seismic building design," in Proceedings of the Structures Congress, pp. 1510-1522, Orlando, Fla, USA, May 2010.

[3] F. Y. Cheng, H. Jiang, and K. Lou, Smart Structures: Innovative Systems for Seismic Response Control, Taylor \& Francis, Boca Raton, Fla, USA, 2008. 
[4] F. Yang, R. Sedaghati, and E. Esmailzadeh, "Optimal design of distributed tuned mass dampers for passive vibration control of structures," Structural Control and Health Monitoring, vol. 22, no. 2, pp. 221-236, 2015.

[5] C. C. Patel and R. S. Jangid, "Seismic response of dynamically similar adjacent structures connected with viscous dampers," The IES Journal Part A: Civil and Structural Engineering, vol. 3, no. 1, pp. 1-13, 2010.

[6] J. T. P. Yao, "Concept of structural control," Structural Division, vol. 98, no. 7, pp. 1567-1574, 1972.

[7] N. S. Xu and Z. H. Yang, "Predictive structural control based on dominant internal model approach," Automatica, vol. 35, no. 1, pp. 59-67, 1999.

[8] H. P. Gavin and U. Aldemir, "Optimal control of earthquake response using semiactive isolation," Journal of Engineering Mechanics, vol. 131, no. 8, pp. 769-776, 2005.

[9] J. N. Yang, "Control of tall buildings under earthquake excitation," Journal of the Engineering Mechanics Division, vol. 108, no. EM5, pp. 883-849, 1982.

[10] J. N. Yang, A. Akbarpour, and P. Ghaemmaghami, "Optimal control algorithms for earthquake excited buildings," in Structural Control: Proceedings of the Second International Symposium on Structural Control, University of Waterloo, Ontario, Canada, July 15-17, 1985, pp. 748-761, Springer, Berlin, Germany, 1987.

[11] O. Bahar, M. R. Banan, M. Mahzoon, and Y. Kitagawa, "Instantaneous optimal Wilson- $\Theta$ control method," Journal of Engineering Mechanics, vol. 129, no. 11, pp. 1268-1276, 2003.

[12] T. T. Soong, Active Structural Control: Theory and Practice, John Wiley \& Sons, New York, NY, USA, 1990.

[13] F. Y. Cheng and P. Tian, "Generalized optimal active control algorithm for nonlinear seismic structures," in Proceedings of the 10th World Conference on Earthquake Engineering, pp. 36773682, Madrid, Spain, 1992.

[14] B. Basu and S. Nagarajaiah, "A wavelet-based time-varying adaptive LQR algorithm for structural control," Engineering Structures, vol. 30, no. 9, pp. 2470-2477, 2008.

[15] B. Basu and S. Nagarajaiah, "Multi scale wavelet-LQR controller for linear time varying systems," Journal of Engineering Mechanics, vol. 136, no. 9, pp. 1143-1151, 2010.

[16] A. Bagheri and F. Amini, "Control of structures under uniform hazard earthquake excitation via wavelet analysis and pattern search method," Structural Control and Health Monitoring, vol. 20, no. 5, pp. 671-685, 2013.

[17] R. Mirzaei and O. Bahar, "A new view on optimal control algorithms," Seismology and Earthquake Engineering, vol. 13, no. 3, pp. 195-207, 2011. 

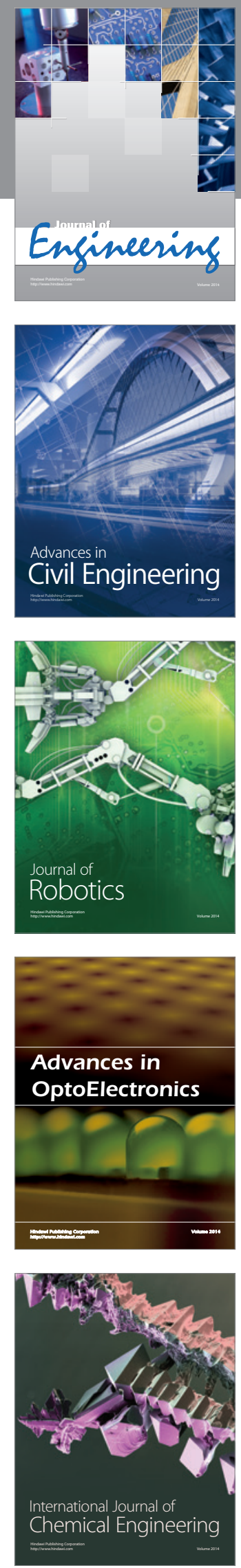

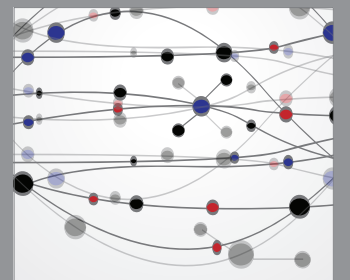

The Scientific World Journal
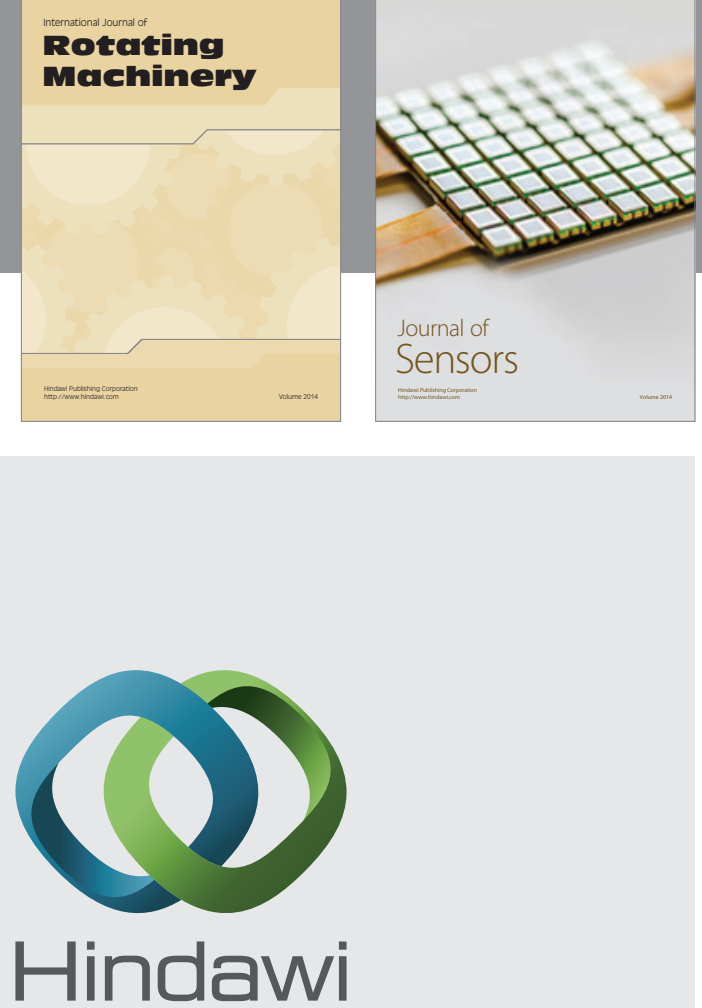

Submit your manuscripts at http://www.hindawi.com
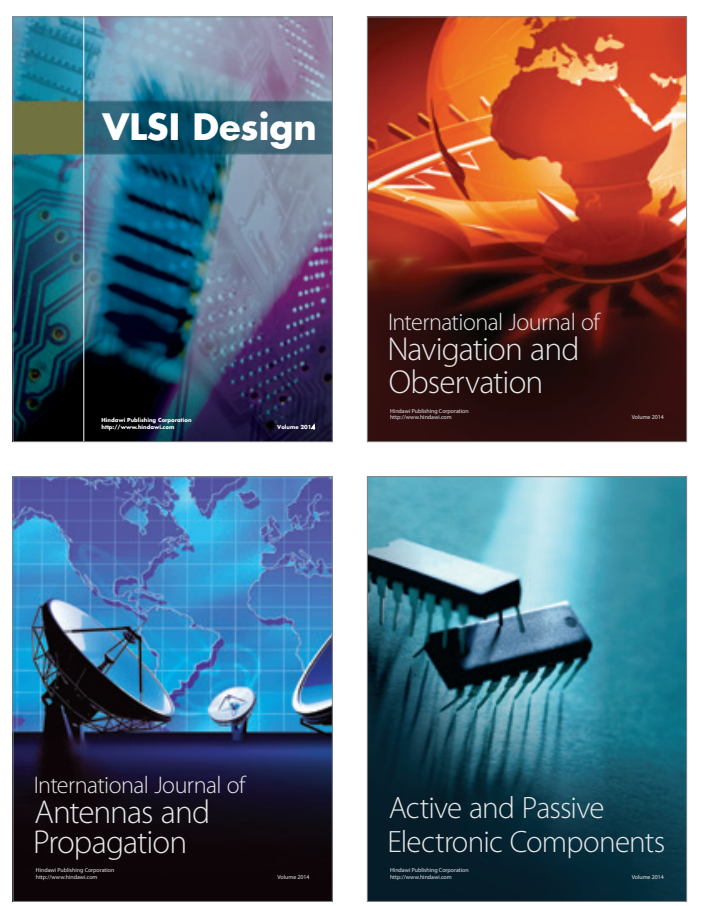
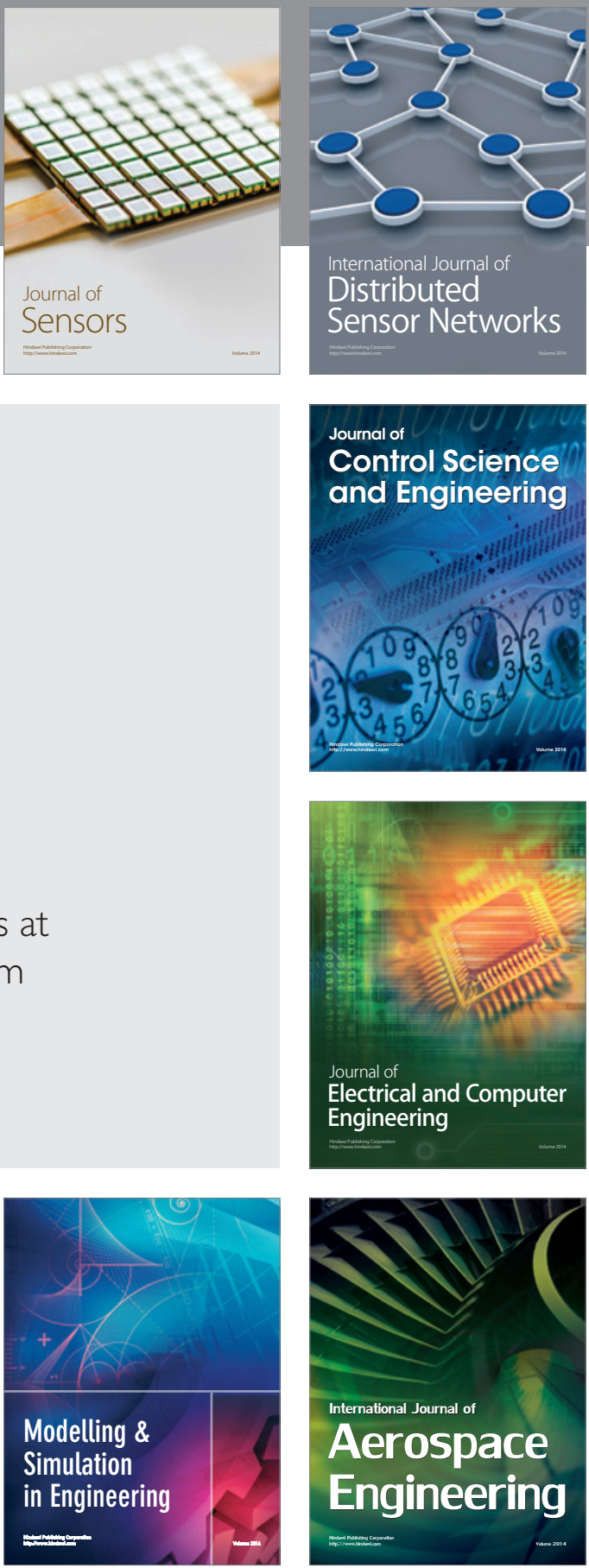

Journal of

Control Science

and Engineering
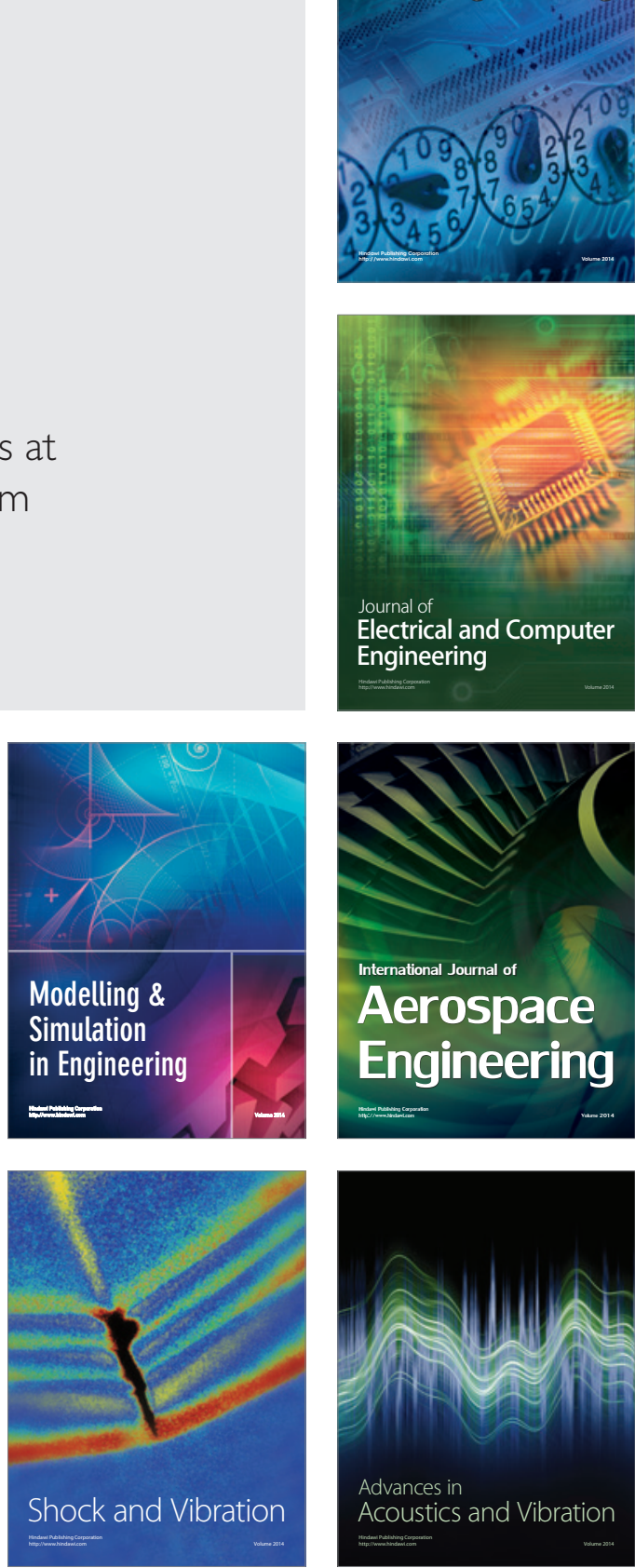